TRANSACTIONS OF THE

AMERICAN MATHEMATICAL SOCIETY

Volume 355, Number 3, Pages 1135-1144

S 0002-9947(02)03129-X

Article electronically published on October 25, 2002

\title{
MAXIMAL FUNCTIONS WITH POLYNOMIAL DENSITIES IN LACUNARY DIRECTIONS
}

\author{
KATHRYN HARE AND FULVIO RICCI
}

\begin{abstract}
Given a real polynomial $p(t)$ in one variable such that $p(0)=0$, we consider the maximal operator in $\mathbb{R}^{2}$,

$$
M_{p} f\left(x_{1}, x_{2}\right)=\sup _{h>0, i, j \in \mathbb{Z}} \frac{1}{h} \int_{0}^{h}\left|f\left(x_{1}-2^{i} p(t), x_{2}-2^{j} p(t)\right)\right| d t .
$$

We prove that $M_{p}$ is bounded on $L^{q}\left(\mathbb{R}^{2}\right)$ for $q>1$ with bounds that only depend on the degree of $p$.
\end{abstract}

\section{INTRODUCTION}

Maximal operators on the real line of the form

$$
f(x) \longmapsto \sup _{h>0} \frac{1}{h} \int_{0}^{h}|f(x-p(t))| d t
$$

where $p$ is a real polynomial with $p(0)=0$, were considered in [CRW1], and it was shown that they satisfy weak-type 1-1 estimates that are uniform over all polynomials of fixed degree. Natural extensions of these operators to higher dimensions are discussed in CRW2, in connection with $\mathbb{R}^{n}$-valued polynomials defined on $\mathbb{R}^{m}$.

We consider here a different kind of multi-dimensional analogue of (1.1), which is modelled on the maximal function in lacunary directions introduced in [NSW]. For simplicity, we restrict ourselves to two dimensions and to dyadic lacunary directions, i.e., determined by the vectors $v_{k}=\left(1,2^{k}\right)$ with $k \in \mathbb{Z}$. In addition, we allow dyadic scaling along each of these directions.

To be precise, given a real polynomial $p(t)$ in one variable such that $p(0)=0$, we define

$$
\begin{aligned}
M_{p} f\left(x_{1}, x_{2}\right) & =\sup _{h>0, i, k \in \mathbb{Z}} \frac{1}{h} \int_{0}^{h}\left|f\left(x-2^{i} p(t) v_{k}\right)\right| d t \\
& =\sup _{h>0, i, j \in \mathbb{Z}} \frac{1}{h} \int_{0}^{h}\left|f\left(x_{1}-2^{i} p(t), x_{2}-2^{j} p(t)\right)\right| d t .
\end{aligned}
$$

We prove the following result.

Theorem 1. $M_{p}$ is bounded on $L^{q}\left(\mathbb{R}^{2}\right)$ for $q>1$ with bounds that only depend on the degree of $p$.

Received by the editors May 27, 2002.

2000 Mathematics Subject Classification. Primary 42B25.

The research of the first author was supported in part by NSERC and the Swedish Natural Sciences Research Council. 
It is easy to check that $M_{p}$ cannot satisfy a weak-type 1-1 estimate.

The proof of Theorem 1 is based on the analysis of a general class of twoparameter maximal operators in the plane defined by compactly supported measures, subject to a decay assumption on their Fourier transforms. This result is in the spirit of $[\mathrm{DR}]$ and $[\mathrm{RS}]$, but here we consider the possibility that the Fourier transform of the measure has no decay within an angle that does not contain the coordinate axes.

Theorem 2. For a probability measure $\mu$ supported on the unit square, let $\mu_{i, j}$ be the measure such that

$$
\int f d \mu_{i, j}=\int f\left(2^{i} x_{1}, 2^{j} x_{2}\right) d \mu\left(x_{1}, x_{2}\right) .
$$

Assume that

(i) there are constants $C, \delta>0$ and $s>1$ such that

$$
|\hat{\mu}(\xi)| \leq C(1+|\xi|)^{-\delta}
$$

away from the set where $s^{-1}<\frac{\left|\xi_{1}\right|}{\left|\xi_{2}\right|}<s$;

(ii) the one-parameter maximal operator

$$
M_{\mu}^{0} f(x)=\sup _{i \in \mathbb{Z}}\left|f * \mu_{i, i}(x)\right|
$$

is bounded on $L^{q}\left(\mathbb{R}^{2}\right)$ for $q>1$.

Then also, the two-parameter maximal operator,

$$
M_{\mu} f(x)=\sup _{i, j \in \mathbb{Z}}\left|f * \mu_{i, j}(x)\right|,
$$

is bounded on $L^{q}\left(\mathbb{R}^{2}\right)$ for $q>1$, with bounds that only depend on $s$, the constants $C, \delta$ in (1.3) and the norm of $M_{\mu}^{0}$.

We start with the proof of Theorem 2, which combines methods from [NSW], [C] and [RS]. This is done in Section 2. Theorem 1 is proved in Section 3.

We thank both W. Beckner and the referee for useful comments on a first version of this paper.

\section{Proof of Theorem 2}

Let $\sigma_{1}$ and $\sigma_{2}$ be the measures on the line defined by

$$
\int_{\mathbb{R}} f(t) d \sigma_{j}(t)=\int_{\mathbb{R}^{2}} f\left(x_{j}\right) d \mu(x) .
$$

Then $\hat{\sigma}_{1}(\tau)=\hat{\mu}(\tau, 0)$ and $\hat{\sigma}_{2}(\tau)=\hat{\mu}(0, \tau)$, so that

$$
\left|\hat{\sigma}_{j}(\tau)\right| \leq C(1+|\tau|)^{-\delta}
$$

Let $\varphi$ be a nonnegative smooth function on the line, supported on $[-1,1]$ and with integral equal to 1 . Define

$$
\nu=\mu-\sigma_{1} \otimes \varphi-\varphi \otimes \sigma_{2}+\varphi \otimes \varphi .
$$

Clearly, $\hat{\nu}$ satisfies (1.3), is supported on the unit square and

$$
\hat{\nu}\left(\xi_{1}, 0\right)=\hat{\nu}\left(0, \xi_{2}\right)=0 \text {. }
$$

Since

$$
M_{\mu} f \leq M_{\nu} f+M_{\sigma_{1} \otimes \varphi} f+M_{\varphi \otimes \sigma_{2}} f+M_{\varphi \otimes \varphi}
$$


we can discuss each of the maximal functions on the right-hand side separately.

The last term is controlled by the two-parameter strong maximal operator of Jessen, Marcinkiewicz and Zygmund. The $L^{q}$-boundedness of the two intermediate terms follows from Theorem 3.2 in [RS], once we observe that, by (2.1),

$$
\left|\widehat{\sigma_{1} \otimes \varphi}(\xi)\right| \leq C^{\prime}(1+|\xi|)^{-\delta},
$$

and similarly for $\varphi \otimes \sigma_{2}$. (Alternatively, one can argue that $M_{\sigma_{1} \otimes \varphi}$ is controlled by the composition of the Hardy-Littlewood maximal operator in the $x_{2}$-variable with the one-parameter operator $M_{\sigma_{1}}$ in the $x_{1}$-variable; to this operator one can apply Theorem A in DR.)

Thus it remains to estimate $M_{\nu} f$. Due to the cancellations of $\nu$ that are implicit in (2.2), it is convenient to introduce appropriate square functions. Given a measure $\sigma$, we shall need two types of such functions:

$$
\begin{array}{r}
S_{\sigma} f(x)=\left(\sum_{i, j \in \mathbb{Z}}\left|f * \sigma_{i, j}(x)\right|^{2}\right)^{\frac{1}{2}}, \\
\tilde{S}_{\sigma} f(x)=\left(\sum_{k \in \mathbb{Z}}\left(\sup _{i \in \mathbb{Z}}\left|f * \sigma_{i, i+k}(x)\right|\right)^{2}\right)^{\frac{1}{2}} .
\end{array}
$$

Clearly, $M_{\sigma} f \leq \tilde{S}_{\sigma} f \leq S_{\sigma} f$. We shall also assume that $q$ is finite, because there is nothing to prove for $q=\infty$.

Let $\eta_{\ell}(x)=2^{2 \ell} \eta\left(2^{\ell} x\right), \ell \geq 0$, be a smooth approximate identity in $\mathbb{R}^{2}$, with $\eta$ supported on the unit disk. We set $\psi_{0}=\eta_{0}$, and $\psi_{\ell}=\eta_{\ell}-\eta_{\ell-1}$ for $\ell \geq 1$. Then

$$
\nu=\sum_{\ell=0}^{\infty} \nu * \psi_{\ell}
$$

and

$$
S_{\nu} f \leq \sum_{\ell=0}^{\infty} S_{\nu * \psi_{\ell}} f
$$

Lemma 2.1. For every $\varepsilon>0$ and $1<q<\infty,\left\|S_{\nu * \psi_{\ell}} f\right\|_{q} \leq A 2^{2 \ell \varepsilon}\|f\|_{q}$, where the constant $A$ depends only on $\varepsilon$ and $q$.

Proof. By the standard randomization argument, we can estimate the $L^{q}$-operator norm of the singular integral operators

$$
f \longmapsto \sum_{i, j} \pm\left(\nu * \psi_{\ell}\right)_{i, j} * f
$$

We apply Lemma 2.3 in $[\mathrm{RS}]$. Thus, it is necessary to prove that

$$
\sup _{0<\left|h_{2}\right|<2}\left|h_{2}\right|^{-\varepsilon} \int\left(\sup _{0<\left|h_{1}\right|<2}\left|h_{1}\right|^{-\varepsilon} \int\left|\Delta_{h_{1}}^{1} \Delta_{h_{2}}^{2}\left(\nu * \psi_{\ell}\right)(x)\right| d x_{1}\right) d x_{2} \leq C 2^{2 \ell \varepsilon}
$$

where

$$
\begin{aligned}
& \Delta_{h_{1}}^{1} f\left(x_{1}, x_{2}\right)=f\left(x_{1}+h_{1}, x_{2}\right)-f\left(x_{1}, x_{2}\right), \\
& \Delta_{h_{2}}^{2} f\left(x_{1}, x_{2}\right)=f\left(x_{1}, x_{2}+h_{2}\right)-f\left(x_{1}, x_{2}\right) .
\end{aligned}
$$

We observe that

$$
\Delta_{h_{1}}^{1} \Delta_{h_{2}}^{2}\left(\nu * \psi_{\ell}\right)=\nu *\left(\Delta_{h_{1}}^{1} \Delta_{h_{2}}^{2} \psi_{\ell}\right)
$$


and that $\Delta_{h_{1}}^{1} \Delta_{h_{2}}^{2} \psi_{\ell}(x)$ is smaller than a constant times $2^{(2+2 \varepsilon) \ell}\left|h_{1}\right|^{\varepsilon}\left|h_{2}\right|^{\varepsilon}$, and it is supported, for each $x, h_{1}, h_{2}$, on a set that is the union of four disks of radius $2^{-\ell}$. Therefore,

$$
\begin{aligned}
\int\left|\Delta_{h_{1}}^{1} \Delta_{h_{2}}^{2}\left(\nu * \psi_{\ell}\right)(x)\right| d x_{1} & \leq \int_{\mathbb{R}^{2}}\left(\int\left|\Delta_{h_{1}}^{1} \Delta_{h_{2}}^{2} \psi_{\ell}(x-y)\right| d x_{1}\right) d|\nu|(y) \\
& \leq C 2^{(1+2 \varepsilon) \ell}\left|h_{1}\right|^{\varepsilon}\left|h_{2}\right|^{\varepsilon} \int_{\mathbb{R}^{2}} \chi_{y, h_{2}}\left(x_{2}\right) d|\nu|(y)
\end{aligned}
$$

where $\chi_{y, h_{2}}$ is the characteristic function of a set of measure $2^{-\ell}$ depending on $y$ and $h_{2}$.

This concludes the proof.

In order to obtain better estimates, we introduce a spectral decomposition of $\nu$. Let $\Phi(\xi)$ be homogeneous of degree 0 , smooth away from the origin, identically equal to 1 inside the angle $\Gamma_{1}=\left\{\xi: s^{-1}<\left|\xi_{1}\right| /\left|\xi_{2}\right|<s\right\}$, and identically equal to 0 outside of the angle $\Gamma_{2}=\left\{\xi:(2 s)^{-1}<\left|\xi_{1}\right| /\left|\xi_{2}\right|<2 s\right\}$.

We then define the "bad part" $\nu_{b}$ of $\nu$ as the distribution such that

$$
\hat{\nu}_{b}(\xi)=\hat{\nu}(\xi) \Phi(\xi)
$$

and the "good part" $\nu_{g}$ as $\nu_{g}=\nu-\nu_{b}$.

The square functions $S_{\nu_{b}} f, S_{\nu_{b} * \psi_{\ell}} f$, etc. are defined as in (2.3.a) and (2.3.b) for Schwartz functions $f$.

We show first that each part of $\nu$ shares the good properties of $\nu$ given by Lemma 2.1 .

Lemma 2.2. The conclusion of Lemma 2.1 remains valid if we replace $\nu$ by $\nu_{b}$ or $\nu_{g}$.

Proof. For $k \in \mathbb{Z}$, let $P_{k} f=\mathcal{F}^{-1}\left(\Phi\left(\xi_{1}, 2^{-k} \xi_{2}\right) \hat{f}(\xi)\right)$. Because of the finite overlapping of the supports of the multipliers $\Phi\left(\xi_{1}, 2^{-k} \xi_{2}\right)$, we have the Littlewood-Paley estimate

$$
\left\|\left(\sum_{k \in \mathbb{Z}}\left|P_{k} f\right|^{2}\right)^{\frac{1}{2}}\right\|_{q} \sim\|f\|_{q},
$$

for $1<q<\infty$. Also, observe that

$$
\left(\nu_{b}\right)_{i, j} * f=\nu_{i, j} *\left(P_{i-j} f\right), \quad\left(\nu_{b} * \psi_{\ell}\right)_{i, j} * f=\left(\nu * \psi_{\ell}\right)_{i, j} *\left(P_{i-j} f\right) .
$$

Therefore,

$$
\begin{aligned}
S_{\nu_{b} * \psi_{\ell}} f & =\left(\sum_{i, j \in \mathbb{Z}}\left|\left(\nu * \psi_{\ell}\right)_{i, j} *\left(P_{i-j} f\right)(x)\right|^{2}\right)^{\frac{1}{2}} \\
& \leq\left(\sum_{i, j, k \in \mathbb{Z}}\left|\left(\nu * \psi_{\ell}\right)_{i, j} *\left(P_{k} f\right)(x)\right|^{2}\right)^{\frac{1}{2}} .
\end{aligned}
$$

The last quantity equals the $L^{2}$-norm on $[0,1]^{3}$ of the function

$$
(t, u, v) \longmapsto \sum_{i, j, k \in \mathbb{Z}}\left(\nu * \psi_{\ell}\right)_{i, j} *\left(P_{k} f\right)(x) r_{i}(t) r_{j}(u) r_{k}(v),
$$


where $r_{n}$ is the $n$th Rademacher function. By the properties of Rademacher functions, the $L^{2}$-norm is equivalent to the $L^{q}$-norm. Therefore,

$\left\|S_{\nu_{b} * \psi_{\ell}} f\right\|_{q}^{q} \leq C \int_{\mathbb{R}^{2}} \int_{[0,1]^{3}}\left|\sum_{i, j, k \in \mathbb{Z}}\left(\nu * \psi_{\ell}\right)_{i, j} *\left(P_{k} f\right)(x) r_{i}(t) r_{j}(u) r_{k}(v)\right|^{q} d t d u d v d x$.

We denote

$$
K_{t, u}=\sum_{i, j} r_{i}(t) r_{j}(u)\left(\nu * \psi_{\ell}\right)_{i, j}, \quad f_{v}=\sum_{k} r_{k}(v) P_{k} f
$$

Changing the order of integration, we have

$$
\left\|S_{\nu_{b} * \psi_{\ell}} f\right\|_{q}^{q} \leq C \int_{[0,1]^{3}}\left\|K_{t, u} * f_{v}\right\|_{q}^{q} d t d u d v .
$$

The proof of Lemma 2.1 shows that the $L^{q}$-operator norms of the $K_{t, u}$ are uniformly bounded by a constant times $2^{2 \ell \varepsilon}$. Hence,

$$
\left\|S_{\nu_{b} * \psi_{\ell}} f\right\|_{q}^{q} \leq C 2^{2 \ell \varepsilon} \int_{[0,1]}\left\|f_{v}\right\|_{q}^{q} d v .
$$

Changing the order of integration again, replacing the $L^{q}$-norm on $[0,1]$ with the $L^{2}$-norm, and using (2.4), we obtain the conclusion for $\nu_{b}$.

For $\nu_{g}$ it is sufficient to observe that $S_{\nu_{g} * \psi_{\ell}} f \leq S_{\nu * \psi_{\ell}} f+S_{\nu_{b} * \psi_{\ell}} f$.

We shall now improve the estimate on $S_{\nu_{g} * \psi_{\ell}}$, using the uniform decay of $\hat{\nu}_{g}(\xi)$ as $\xi$ goes to infinity. In fact, as we already observed, $\hat{\nu}$ satisfies (1.3); hence,

$$
\left|\hat{\nu}_{g}(\xi)\right| \leq C(1+|\xi|)^{-\delta} .
$$

We shall assume, w.l.o.g., that $\delta<1$.

Lemma 2.3. $\left\|S_{\nu_{g} * \psi_{\ell}} f\right\|_{2} \leq A 2^{-\ell \delta / 4}\|f\|_{2}$, with $A$ depending only on $\delta$ and $C$.

Proof. By the Plancherel formula, we have to prove that

$$
\sum_{i, j \in \mathbb{Z}}\left|\hat{\nu}_{g}\left(2^{i} \xi_{1}, 2^{j} \xi_{2}\right)\right|^{2}\left|\hat{\psi}_{\ell}\left(2^{i} \xi_{1}, 2^{j} \xi_{2}\right)\right|^{2} \leq A 2^{-\ell \delta / 2} .
$$

By $(2.2)$,

$$
\hat{\nu}(\xi)=\int\left(e^{-i x_{1} \xi_{1}}-1\right)\left(e^{-i x_{2} \xi_{2}}-1\right) d \nu(\xi) .
$$

Since $\nu$ is supported on the unit square,

$$
|\hat{\nu}(\xi)| \leq C\left|\xi_{1}\right|\left|\xi_{2}\right|
$$

Combining this with (2.5), we obtain that, if $0<\varepsilon<1$,

$$
\left|\hat{\nu}_{g}(\xi)\right| \leq C \frac{\left|\xi_{1}\right|^{\varepsilon}\left|\xi_{2}\right|^{\varepsilon}}{(1+|\xi|)^{\delta(1-\varepsilon)}}
$$

If $\ell \geq 1$, then

$$
\left|\hat{\psi}_{\ell}(\xi)\right|=\left|\hat{\psi}_{1}\left(2^{-(\ell-1)} \xi\right)\right| \leq C 2^{-\ell \varepsilon}|\xi|^{\varepsilon},
$$

because $\hat{\psi}_{1}(0)=0$. Hence,

$$
\left|\hat{\nu}_{g}(\xi) \hat{\psi}_{\ell}(\xi)\right| \leq C 2^{-\ell \varepsilon} \frac{\left|\xi_{1}\right|^{\varepsilon}\left|\xi_{2}\right|^{\varepsilon}}{(1+|\xi|)^{\delta(1-\varepsilon)-\varepsilon}}
$$


We can assume that $\left|\xi_{1}\right| \sim\left|\xi_{2}\right| \sim 1$ in (2.6). Then we simply have to observe that, taking $\varepsilon=\delta / 4$, the exponent in the denominator is bigger than $\delta / 2=2 \varepsilon$, and that the series

$$
\sum_{i, j \in \mathbb{Z}} \frac{2^{2 \varepsilon i} 2^{2 \varepsilon j}}{\left(1+2^{i}+2^{j}\right)^{2 \alpha}}
$$

is convergent for $\alpha>2 \varepsilon$.

Interpolating between the $L^{2}$-estimate in Lemma 2.3 and the $L^{q}$-estimate in Lemma 2.2 for $S_{\nu_{g} * \psi_{\ell}}$, we obtain that for every $q \in(1, \infty)$ there is an $\varepsilon_{q}>0$ such that $\left\|S_{\nu_{g} * \psi_{\ell}} f\right\|_{q} \leq A 2^{-\ell \varepsilon_{q}}\|f\|_{q}$. Therefore,

Proposition 2.4. $S_{\nu_{g}}$ is bounded on $L^{q}$ for $1<q \leq 2$.

In order to complete the proof of Theorem 2, we may just observe that we are in the hypotheses of Theorem B in [C] (attributed to M. Christ). We give, however, an independent proof, based on the extrapolation argument in [NSW], adapted to $\tilde{S}_{\nu_{b}}$.

End of the proof of Theorem 2. The starting point is that $\tilde{S}_{\nu_{b}}$ is bounded on $L^{2}$. In fact, assumption (ii) implies that $M_{\nu_{0, k}}^{0}$ is uniformly bounded on $L^{q}$ independently of $k$. Therefore,

$$
\begin{aligned}
\int \tilde{S}_{\nu_{b}} f(x)^{2} d x & =\sum_{k \in \mathbb{Z}} \int \sup _{i \in \mathbb{Z}}\left|\nu_{i, i+k} * P_{k} f(x)\right|^{2} d x \\
& =\sum_{k \in \mathbb{Z}} \int\left(M_{\nu_{0}, k}^{0} P_{k} f(x)\right)^{2} d x \\
& \leq C \sum_{k \in \mathbb{Z}} \int\left(P_{k} f(x)\right)^{2} d x \\
& =C\|f\|_{2}^{2} .
\end{aligned}
$$

In general, the boundedness of $\tilde{S}_{\nu_{b}}$ on some $L^{q}$ implies, by Proposition 2.4, the boundedness of $M_{\nu}$ on the same $L^{q}$, and hence that of $M_{\mu}$.

Assume now that $M_{\mu}$ is bounded on some $L^{q}$, and consider the inequality

$$
\left\|\left(\sum_{k \in \mathbb{Z}} M_{\mu_{0, k}}^{0} f_{k}(x)^{r}\right)^{1 / r}\right\|_{s} \leq C\left\|\left(\sum_{k \in \mathbb{Z}}\left|f_{k}(x)\right|^{r}\right)^{1 / r}\right\|_{s} .
$$

This is equivalent to saying that the linear operator

$$
T:\left\{f_{k}\right\} \longmapsto\left\{\mu_{i, i+k} * f_{k}\right\}
$$

is bounded from $L^{s}\left(\ell^{r}\right)$ to $L^{s}\left(\ell^{r}\left(\ell^{\infty}\right)\right)$.

Since $\mu$ is a positive measure and we are assuming that $M_{\mu}$ is bounded on $L^{q}$, (2.7) is verified for $r=\infty$ and $s=q$. In addition, it is verified for $r=s>1$ by the uniform boundedness of $M_{\mu_{0, k}}^{0}$. Hence, $T$ is bounded from $L^{q}\left(\ell^{\infty}\right)$ to $L^{q}\left(\ell^{\infty}\left(\ell^{\infty}\right)\right)$ and from $L^{r}\left(\ell^{r}\right)$ to $L^{r}\left(\ell^{r}\left(\ell^{\infty}\right)\right)$ for $r>1$. By interpolation, (2.7) holds for $r=2$ and $\frac{1}{q}<\frac{1}{s}<\frac{1}{2}\left(1+\frac{1}{q}\right)$. 
The same inequality holds with $\mu$ replaced by $\sigma \otimes \varphi, \varphi \otimes \sigma$ and $\varphi \otimes \varphi$, and hence with $\mu$ replaced by $\nu$.

Taking $f_{k}=P_{k} f$, this implies that $\tilde{S}_{\nu_{b}}$ is bounded on the same spaces $L^{s}$. Since each $q \in(1,2)$ can be reached by iteration in a finite number of steps, we conclude that $M_{\mu}$ is bounded on $L^{q}$ for every $q>1$.

\section{Proof of Theorem 1}

The starting point for the proof of Theorem 1 is Lemma 2.5 in [CRW1]. We give a slightly different (and less complete) formulation of it.

Lemma 3.1. For every $n$ there are constants $A(n) \geq 1$ and $B=B(n)$ with the following property: if $p(t)$ is a monic real polynomial of degree $n$ such that $p(0)=0$, $A \geq A(n)$, and $m \in \mathbb{Z}$ is such that no complex zero of $p$ lies in the strip

$$
\left\{z: A^{m-1} \leq|z| \leq A^{m+2}\right\},
$$

then the following properties hold:

(i) $p$ has constant sign and is strictly monotonic on $I_{m}=\left[A^{m}, A^{m+1}\right]$;

(ii) $|p(t)| \leq B t\left|p^{\prime}(t)\right|$ for $t \in I_{m}$;

(iii) $\max _{t \in I_{m}}|p(t)| \leq B \min _{t \in I_{m}}|p(t)|$.

Observe that we are allowed to replace the polynomial $p(t)$ in $(1.2)$, when convenient, by $\tilde{p}(t)=b p(a t)$, with $a, b>0$. In fact, the identity

$$
M_{\tilde{p}} f(x)=M_{p} f_{b}\left(\frac{x}{b}\right),
$$

where $f_{b}(x)=f(b x)$, implies that $M_{p}$ and $M_{\tilde{p}}$ have the same operator norm. In particular, we can assume that $p$ is monic.

Also, the maximal function $M_{p}$ can be replaced by

$$
\tilde{M}_{p} f\left(x_{1}, x_{2}\right)=\sup _{m \in \mathbb{Z}} \tilde{M}_{p, m} f\left(x_{1}, x_{2}\right)
$$

where

$$
\tilde{M}_{p, m} f\left(x_{1}, x_{2}\right)=\sup _{i, j \in \mathbb{Z}} A^{-m} \int_{I_{m}}\left|f\left(x_{1}-2^{i} p(t), x_{2}-2^{j} p(t)\right)\right| d t .
$$

Let $I_{m}$ be one of the "good" dyadic intervals satisfying properties (i)-(iii) in Lemma 3.1. Making the change of variable $u=p(t)$, we have

$$
\begin{aligned}
A^{-m} \int_{I_{m}} \mid f\left(x_{1}-2^{i} p(t),\right. & \left.x_{2}-2^{j} p(t)\right) \mid d t \\
& \leq A \int_{A^{m}}^{A^{m+1}}\left|f\left(x_{1}-2^{i} p(t), x_{2}-2^{j} p(t)\right)\right| \frac{d t}{t} \\
& \leq A B \int_{I_{m}}\left|f\left(x_{1}-2^{i} p(t), x_{2}-2^{j} p(t)\right)\right| \frac{\left|p^{\prime}(t)\right|}{|p(t)|} d t \\
& =A B \int_{p\left(I_{m}\right)}\left|f\left(x_{1}-2^{i} u, x_{2}-2^{j} u\right)\right| \frac{d u}{|u|} .
\end{aligned}
$$

By (i) and (iii), the interval $p\left(I_{m}\right)$ is contained in an interval of the form $\pm\left[\alpha_{m}, B \alpha_{m}\right]$, with $\alpha_{m}>0$. Therefore, assuming w.l.o.g. that $p$ is positive on $I_{m}$ 


$$
\begin{aligned}
A^{-m} \int_{I_{m}} \mid f\left(x_{1}-2^{i} p(t),\right. & \left.x_{2}-2^{j} p(t)\right)\left|d t \leq A B \int_{\alpha_{m}}^{B \alpha_{m}}\right| f\left(x_{1}-2^{i} u, x_{2}-2^{j} u\right) \mid \frac{d u}{u} \\
& \leq \frac{A B}{\alpha_{m}} \int_{\alpha_{m}}^{B \alpha_{m}}\left|f\left(x_{1}-2^{i} u, x_{2}-2^{j} u\right)\right| d u \\
& \leq \frac{A B^{2}}{B \alpha_{m}} \int_{0}^{B \alpha_{m}}\left|f\left(x_{1}-2^{i} u, x_{2}-2^{j} u\right)\right| d u
\end{aligned}
$$

This shows that the contribution to $\tilde{M}_{p} f$ given by the "good" intervals is controlled by the maximal function in lacunary directions

$$
\mathcal{M} f\left(x_{1}, x_{2}\right)=\sup _{h>0, k \in \mathbb{Z}} \frac{1}{h} \int_{0}^{h}\left|f\left(x_{1}-t, x_{2}-2^{k} t\right)\right| d t
$$

of [NSW]. Since $\mathcal{M}$ is bounded on $L^{q}$ for $q>1$ [NSW], it remains to consider the contribution from the "bad" intervals. Since there are at most $3 n$ of these intervals, it is enough to prove that $\tilde{M}_{p, m}$ acts on $L^{q}$ for $q>1$, with operator norm bounded independently of the polynomial $p$ and integer $m$.

We claim it suffices to show that there exists a constant $C_{q, n}$ such that

$$
\sup _{m \in \mathbb{Z}}\left\|\tilde{M}_{p, m} f\right\|_{q} \leq C_{q, n}\|f\|_{q}
$$

for every $f \in L^{q}$ and monic polynomial $p$ of degree $n$ satisfying $p(0)=0$ and

$$
A^{-n} \leq \max _{t \in I_{m}}|p(t)| \leq 1
$$

To see this, suppose $p$ is an arbitrary monic polynomial with $p(0)=0$, and choose $k \in \mathbb{Z}$ such that

$$
A^{-n} \leq \max _{t \in I_{m}} A^{-k n}|p(t)| \leq 1
$$

Let $\underset{\sim}{p}(t)=A^{-k n} p\left(A^{k} t\right)$. Since

$$
A^{-n} \leq \max _{t \in I_{m-k}}|p(t)| \leq 1
$$

the $\left(L^{q}, L^{q}\right)$ operator norm of $\tilde{M}_{p, m-k}$ is at most $C_{q, n}$. Since

$$
\tilde{M}_{p, m} f(x)=\tilde{M}_{\sim}, m-k f_{A^{k}}\left(A^{-k} x\right)
$$

$\tilde{M}_{p, m}$ also acts on $L^{q}$ with bounds that are independent of $m$ and $p$.

Consequently, we need to investigate the measure $\mu$ given by

$$
\int f d \mu=A^{-m} \int_{I_{m}} f(p(t), p(t)) d t
$$

where $p$ satisfies (3.2). This measure is supported on the segment $\{(u, u):-1 \leq$ $u \leq 1\}$ and, up to a factor depending on $A$, is a probability measure.

The proof of Theorem 1 will be complete once we show that the operator $M_{\mu}$ is bounded on $L^{q}$ for $q>1$ with bounds that depend only on $n$ and $q$. We apply Theorem 2.

The Fourier transform of $\mu$ is

$$
\hat{\mu}\left(\xi_{1}, \xi_{2}\right)=A^{-m} \int_{I_{m}} e^{-i\left(\xi_{1}+\xi_{2}\right) p(t)} d t .
$$


Lemma 3.2. There is an integer $k \in\{1,2, \ldots, n\}$ such that, if $A$ is large enough (depending on $n$ ), then

$$
\left|\hat{\mu}\left(\xi_{1}, \xi_{2}\right)\right| \leq C A^{n}\left(1+\left|\xi_{1}+\xi_{2}\right|\right)^{-1 / k}
$$

with $C$ independent of $p$ and $m$.

Proof. Let $t_{1}=0, t_{2}, \ldots, t_{n}$ be the zeroes of $p$, ordered so that $0 \leq\left|t_{2}\right| \leq \cdots \leq\left|t_{n}\right|$. Let $m^{\prime}$ be the smallest integer greater than $m$ such that $I_{m^{\prime}}$ does not contain any of the $\left|t_{j}\right|$. Then $m^{\prime} \leq m+n$, so that $A^{m^{\prime}}$ is comparable with $A^{m}$. Also let $k$ be such that $\left|t_{j}\right|<A^{m^{\prime}}$ for $j \leq k$ and $\left|t_{j}\right|>A^{m^{\prime}+1}$ for $j>k$.

The $k$ th derivative of $p$ equals

$$
p^{(k)}(t)=\prod_{j=k+1}^{n}\left(t-t_{j}\right)+r(t)
$$

where $r(t)$ is a sum where each term is a product of $n-k$ factors $t-t_{j}$, with at least one of the $j$ less than or equal to $k$.

If $t \in I_{m},\left|t-t_{j}\right|<2 A^{m^{\prime}}$ for $j \leq k$, and $\left|t-t_{j}\right|>\left(1-A^{-1}\right)\left|t_{j}\right|>(A-1) A^{m^{\prime}}$ for $j>k$. Therefore, if $A$ is large enough,

$$
\left|p^{(k)}(t)\right| \geq C \prod_{j=k+1}^{n}\left|t_{j}\right|,
$$

for $t \in I_{m}$.

By van der Corput's lemma,

$$
A^{-m}\left|\int_{I_{m}} e^{-i \lambda p(t)} d t\right| \leq C A^{-m}\left(\prod_{j=k+1}^{n}\left|t_{j}\right|\right)^{-1 / k}|\lambda|^{-1 / k} .
$$

If $\bar{t} \in I_{m}$ is such that $|p(\bar{t})| \geq A^{-n}$, we have

$$
A^{-n} \leq|p(\bar{t})| \leq 2^{n} A^{k m^{\prime}} \prod_{j=k+1}^{n}\left|t_{j}\right| .
$$

Therefore, $\prod_{j=k+1}^{n}\left|t_{j}\right| \geq C A^{-n} A^{-k m}$, so that

$$
A^{-m}\left|\int_{I_{m}} e^{-i \lambda p(t)} d t\right| \leq C|\lambda|^{-1 / k} A^{n}
$$

with $C$ independent of $p$ and $m$. Since the left-hand side is trivially bounded by 1 , this concludes the proof.

Thus, $\hat{\mu}$ clearly satisfies hypothesis (i) of Theorem 2 . It remains to prove that the one-parameter maximal operator $M_{\mu}^{0}$ in (1.4) is bounded on $L^{q}$ for $q>1$ with bounds that only depend on $n$ and $q$. This follows from a transference argument: because $\mu$ is supported on a line, it is sufficient to consider the maximal operator on $\mathbb{R}$,

$$
M_{\tilde{\mu}} g(x)=\sup _{i \in \mathbb{Z}}\left|g * \tilde{\mu}_{i}(x)\right|,
$$

where

$$
\int_{\mathbb{R}} g d \tilde{\mu}=A^{-m} \int_{I_{m}} g(p(t)) d t .
$$

By Lemma 3.2, $|\widehat{\tilde{\mu}}(\eta)| \leq C(1+|\eta|)^{-1 / k}$, with $1 \leq k \leq n$ and $C$ depending only on $n$. The conclusion follows from Theorem $\mathrm{A}$ in $[\mathrm{DR}$. 
Remark. In [CRW1] the authors show that the "supermaximal function" on the real line

$$
f(x) \longmapsto \sup _{\substack{h>0 \\ \operatorname{deg} p \leq k, p(0)=0}} \frac{1}{h} \int_{0}^{h}|f(x-p(t))| d t
$$

is of restricted weak type $k-k$ and hence of strong type $q-q$ for $q>k$.

The proof can be adapted to show that the operator

$$
\begin{aligned}
\mathcal{M}_{k} f(x) & =\sup _{\operatorname{deg} p \leq k, p(0)=0} M_{p} f(x) \\
& =\sup _{\substack{h>0, j \in \mathbb{Z} \\
\operatorname{deg} p \leq k, p(0)=0}} \frac{1}{h} \int_{0}^{h}\left|f\left(x_{1}-p(t), x_{2}-2^{j} p(t)\right)\right| d t
\end{aligned}
$$

is bounded on $L^{q}\left(\mathbb{R}^{2}\right)$ for $q>k$.

In fact, if $f$ is the characteristic function of a measurable set in the plane, the same proof as in CRW1 gives the pointwise domination

$$
\mathcal{M}_{k} f(x) \leq C\left(M^{*} f^{k}(x)\right)^{1 / k},
$$

where $M^{*}$ is the maximal function in dyadic direction of [NSW]. This implies that $\mathcal{M}_{k}$ is of restricted weak type $q-q$ for $q>k$, and hence of strong type.

\section{REFERENCES}

[C] A. Carbery, Differentiation in lacunary directions and an extension of the Marcinkiewicz multiplier theorem, Ann. Inst. Fourier (Grenoble) 38 (1988), 157-168. MR 89h:42026

[CRW1] A. Carbery, F. Ricci, and J. Wright, Maximal functions and Hilbert transforms associated to polynomials, Rev. Mat. Iberoam. 14 (1998), 117-144. MR 99k:42014

[CRW2] A. Carbery, F. Ricci, and J. Wright, Maximal functions and singular integrals associated to polynomial mappings of $\mathbb{R}^{n}$, preprint.

[DR] J. Duoandikoetxea and J. L. Rubio de Francia, Maximal and singular integral operators via Fourier transform estimates, Invent. Math. 84 (1986), 541-561. MR 87f:42046

[NSW] A. Nagel, E. M. Stein and S. Wainger, Differentiation in lacunary directions, Proc. Natl. Acad. Sci. U.S.A. 75 (1978), 1060-1062. MR 57:6349

[RS] F. Ricci and E. M. Stein, Multiparameter singular integrals and maximal functions, Ann. Inst. Fourier (Grenoble) 42 (1992), 637-670. MR 94d:42020

Department of Pure Mathematics, University of Waterloo, Waterloo, Ontario, CANADA, N2L 3G1

E-mail address: kehare@math.uwaterloo.ca

Scuola Normale Superiore, Piazza dei Cavalieri 7, 56126 Pisa, Italy

E-mail address: fricci@sns.it

URL: http://www.math.sns.it/HomePages/Ricci/ 